\title{
Pathogens Isolated in Respiratory Tract Samples of Recently Hospitalized Patients: Implications for Primary Care Physicians
}

\section{Oryan Henig}

Rambam Health Care Campus

Anat Reiner Benaim

Rambam Health Care Campus

Ami Neuberger ( $\nabla$ a_neuberger@rambam.health.gov.il )

Rambam Health Care Campus

\section{Research article}

Keywords: Lower respiratory tract infection, Pneumonia, Microbiology, Pathogens, Community, Antibiotics, Drug resistance, Bacterial resistance, Epidemiology

Posted Date: July 24th, 2020

DOI: https://doi.org/10.21203/rs.3.rs-46002/v1

License: () (i) This work is licensed under a Creative Commons Attribution 4.0 International License. Read Full License 


\section{Abstract \\ Background.}

Little is known about the etiology of community-acquired lower respiratory tract infections (CA-LRTI), and treatment is largely empirical. We aimed to provide clinicians with microbiologic data of the more severe cases, i.e. those that result in hospital admission.

\section{Methods.}

A retrospective observational cohort study was conducted at a tertiary care hospital in Haifa, Israel. Consecutive respiratory tract samples obtained from admitted patients older than 12 years between 2014 and 2020 were included. Pathogen distribution and drug susceptibility were described, and factors associated with 14 day mortality rates were analyzed using a multivariable logistic regression with a stepwise model reduction.

\section{Results.}

A total of 1,395; 2,212; and 2,760 samples were included in the community-acquired, healthcare-associated and hospital-acquired LRTI groups, respectively. Gram negative bacteria were the most common bacteria isolated. Streptococcus pneumoniae was over represented in patients admitted to the intensive care unit (ICU) with CA-LRTI and healthcare-associated LRTI (accounting for $20 \%$ and $10.1 \%$ of patients admitted to the ICU versus $12.6 \%$ and $6.4 \%$ of patients in the entire cohort, $\mathrm{p}<0.001$ ). Streptococcus pneumoniae was susceptible to penicillin in around $50 \%$ of cases; to erythromycin in $65 \%$ of cases; and to fluoroquinolones and 3rd generation cephalosporins in more than $95 \%$ of cases. Twenty-five percent of other typical community-acquired pathogens (Hemophillus influenza, Moraxella spp., and Bordatella spp.) were resistant to ampicillin. Susceptibility of Gram negative bacteria to penicillins with and without a $\beta$-lactamase inhibitor and to second generation cephalosporins was lower than $50 \%$. In the multivariable analysis, factors that were associated with increased mortality in the entire cohort were: age, admission to an ICU or surgical department, healthcare-associated or hospital-acquired infections, and infections with gram negative bacteria, Staphylococcus aureus, and Stenotrophomonas or Acinetobacter baummannii.

\section{Conclusions.}

Our data shows that gram negative bacteria are common causative agents among elderly patients with multiple comorbidities. Streptococcus pneumoniae and other typical community acquired pathogens are proportionally more common in younger patients, although most absolute cases occur in elderly patients. The susceptibility pattern of these community pathogens suggests that for high-risk patients the choice of effective oral antibiotic agents in the community is limited.

\section{Background}

A community-acquired lower respiratory tract infection (CA-LRTI) is one of the most common infections attended by primary care physicians and results in considerable use of antibiotics [1, 2]. As microbiological analysis of sputum cultures is rarely performed in community settings, little is known about the etiology of CA-LRTI, and bacterial susceptibilities of causative pathogens are unknown in most areas. Treatment of LRTI by primary care physicians is thus largely empirical with penicillins, 2nd and 3rd generation cephalosporins, fluoroquinolones, doxycycline and macrolides often used. On the one hand, such empirical treatment may be superfluous resulting in more antibiotic resistance in the community [3]. On the other hand, for the few patients whose treatment fails and results in eventual hospital admission, antibiotic treatment may be inadequate.

In the few studies that have examined the microbiology of community acquired pneumonia among relatively young patients, the only culturable pathogens that were found in a significant proportion of cases (i.e. > 5\%) are Streptococcus pneumoniae and Hemophillus influenza [4]. Other pathogens, such as Moraxella spp., Bordatella spp. and Staphylococcus aureus are uncommon [5]. Whereas, common pathogens such as Legionella pneuumophila, Mycoplasma pneumoniae or Chlamydia pneumoniae are only diagnosed through molecular methods or serological tests [6]. Gram negative bacteria are detected in $2.8 \%-14 \%$ of community acquired pneumonia cases, particularly in the elderly and patients who are admitted from a long-term care facility (LTCF) [7, 8].

By analyzing the results of respiratory tract cultures of recently admitted patients, we aimed to provide physicians with the relevant microbiologic data of the more severe CA-LRTI cases, i.e. those that resulted in hospital admission. Such microbiological data would provide primary care and emergency room physicians with additional insights as to the causative agents of severe CA-LRTI, thereby improving the process of selecting empirical antibiotic therapy for patients with high-risk LRTI in the community.

\section{Methods}

\section{Study Setting and Cohort Description}

A retrospective observational cohort study was performed at the Rambam Health Care Campus (RHCC), a 970-bed primary and tertiary care hospital in Haifa, Israel.

The cohort included all consecutive respiratory tract samples obtained from patients older than 12 years who were admitted to the RHCC between January 2014 and January 2020. The Institutional Review Board of the RHCC approved the study for waiver of informed consent. 


\section{Data Collection}

For each patient's admission throughout the study period, all unique bacterial species were included (i.e. the first bacteria of each species for each admission). Data were retrieved with the use of the institution's electronic medical records. Data pertaining to demographics, source of admission (home, LTCF), hospitalization at the RHCC within the past 90 days, Charlson Comorbidity Index (CCI), and admission unit were collected for each admission. Microbiology data included dates of sample collection, dates of results, name of bacteria species, and antimicrobial susceptibility.

\section{Outcome Definitions}

Bacterial pathogens were divided into seven groups: 1) Enterobacteriacea; 2) Gram negative bacteria associated with community-acquired infections Hemophillus influenza, Moraxella and Bordatella spp.; 3) non-fermenting gram negative bacteria - Pseudomonas and Burkholderia spp.; 4) Other gram negative bacteria - Serratia, Providencia, and Proteus spp.; 5) Staphylococcus aureus, 6) Opportunistic bacteria common among intensive care unit (ICU) patients - Stenotrophomonas and Acinetobacter spp.; and 7) Streptococcus pneumoniae.

We defined a group of pathogens as 'typical community pathogens' based on previous large studies that showed Streptococcus pneumoniae and Hemophillus influenza to be by far the most common bacterial pathogens to cause CA-LRTI [4]. Moraxella and Bordatella spp., although uncommon, were also included in this group as they rarely cause hospital-acquired pulmonary infections $[9,10]$.

Drug resistance was defined according to the Clinical and Laboratory Standards Institute (CLSI) breakpoints for susceptibility. Bacteria with intermediate drug susceptibility were defined as resistant. Finally, fourteen-day mortality was assessed in relation to the date the index culture was obtained.

\section{Variable Definitions}

The place of acquisition of each culture was classified with the use of three factors: 1) the time elapsed from date of admission to the date the sample was collected; 2) whether the admission was from a LTCF; and 3) if the patient had been hospitalized within the past 3 months. Accordingly, the study consisted of three cohorts as follows: i) Community-acquired cultures which were defined as cultures obtained within less than 48 hours of admission of patients who were not residing in an LTCF and were not hospitalized in the RHCC within the past 3 months; ii) Healthcare-associated (but community-acquired) cultures which were defined as cultures collected within 48 hours of admission of patients who were either admitted from an LTCF or hospitalized in the RHCC within the past 3 months; and iii) Hospital-acquired cultures which were defined as cultures collected from patients who were hospitalized for no less than 3 days but for no more than 7 days.

The patients' ages at the time of culture collection were stratified into 3 groups as follows: $12-18$ years; $>18$ years to 40 years; and older than 40 years. The CCl was stratified into 3 groups: 1 ) a CCl of $0 ; 2$ ) a CCl between 1 and 4 ; and 3 ) a $\mathrm{CCl}$ of $>4$.

Referring wards were stratified into 5 groups: 1) Internal medicine and Emergency; 2) Pediatrics; 3) ICU; 4) Oncology and Hemato-oncology; and 5) Surgery and Orthopedics.

\section{Statistical Analysis}

Continuous variables were described with the use of median and $25-75 \%$ interquartile ranges (IQR). We analyzed factors associated with a 14 -day mortality in the entire cohort, and in the cohort of patients with typical community pathogens (Streptococcus pneumoniae, Hemophillus influenza, Bordatella spp., Moraxella spp.) using a multivariate logistic regression with a stepwise model reduction, and accounting for age, gender, referring ward, and place of acquisition. A p-value of 0.05 was considered statistically significant.

\section{Results}

A total of 1,395; 2,212; and 2,760 samples were included in the community-acquired, healthcare-associated and hospital-acquired LRTI groups, respectively. Of the 2,212 healthcare-associated samples, 295 were obtained from patients coming from LTCFs, while 1917 were obtained from patients with previous hospital admissions. Samples taken from female patients accounted for 1884 / 6367 (29.6\%) of the entire cohort. The mean age was $60.6 \pm 19.9$ years (median 65 , IQR = 50-75), and the mean CCI was $5.7 \pm 4.1$ (median 5, IQR = 2-8.5). Samples were obtained in the pediatric ward $(267,4.2 \%)$; ICU (1823, 28.6\%); emergency room and internal medicine wards (3190, 50.1\%); oncology and hemato-oncology wards (190, 2.5\%); and surgery and orthopedics wards (897, 14.1\%). Sputum samples, tracheal aspirations or broncho-alveolar lavage were used in 3912 (61.4\%), 1879 (29.5\%), and 576 (9\%) of all specimens, respectively. These demographic data are presented in Table 1. 
Table 1

Characteristics of patients with LRTI

\begin{tabular}{|ll|}
\hline & Entire cohort (N = 6367 samples) \\
\hline Age (Median, IQR) & $65(50,75)$ \\
\hline Female (N (\%)) & $1884(30)$ \\
\hline Charlson comorbidity index (Median, IQR) & $5(2,8.5)$ \\
\hline Place of acquisition & \\
\hline Community (N (\%)) & $1395(21.9)$ \\
\hline Healthcare associated (N (\%)) & $2212(34.7)$ \\
\hline Hospital acquired (N (\%)) & $2760(43.3)$ \\
\hline Department & $267(4.2)$ \\
\hline Pediatric (N (\%)) & $3190(50.1)$ \\
\hline Internal medicine (N (\%)) & $897(14.1)$ \\
\hline Surgery-orthopedic (N (\%)) & $190(2.5)$ \\
\hline Hemato-oncology (N (\%)) & $1823(28.6)$ \\
\hline ICU (N (\%)) & \\
\hline Source of specimen & $3912(61.4)$ \\
\hline Sputum (N (\%)) & $1879(29.5)$ \\
\hline Tracheal aspiration (N (\%)) & \\
\hline Broncho-alveolar lavage (N (\%)) & \\
\hline LRTI, lower respiratory tract infection & \\
\hline
\end{tabular}

Pathogen distribution among the three study cohorts is shown in Table 2. The probability of isolating bacteria which we initially expected to be "typical" community-acquired pathogens, namely Streptococcus pneumoniae, Hemophillus influenza, Bordatella spp., Moraxella spp., was higher among patients with CA-LRTI ( $p<0.0001$ for all comparisons). Enterobacteriacea, non-fermenting gram negative bacteria, and other gram negative bacteria, were more frequently isolated from patients with healthcare-associated and hospital-acquired LRTI, but were by far the most common pathogens isolated in all cohorts ( $<<0.0001$ for all comparisons), while Staphylococcus aureus was relatively equally distributed among the three study cohorts. These observations were also true when patients were divided into age groups (12-18 years, > 18-40 years, > 40 years; Fig. 1), or according to the number of comorbid conditions included in the CCI (Fig. 2).

Table 2

Pathogen distribution among patients with community-acquired, healthcare-associated and hospital-acquired LRTI



Overall, the number of respiratory cultures was higher among patients older than 40 years compared to other age groups. The relative proportion of samples with a growth of Streptococcus pneumoniae was significantly higher $(p<0.001)$ among patients aged 18 to 40 years $(9.8 \%$ of all samples) when compared to younger patients (7.1\%), or to adults aged more than 40 years $(5.9 \%)$, although the absolute number of samples positive for this bacterium was much higher 
among older patients. Similarly, Hemophillus influenza, Moraxella spp., and Bordatella spp., were isolated in $15.1 \%, 18.1 \%$ and $12.6 \%$ of samples from patients aged 12 to 18 years, $>18$ to 40 , and $>40$ years, respectively $(p<0.001$ for all comparisons), but the vast majority of samples positive for these bacteria were taken from patients older than 40 years.

Pathogen distribution in samples taken from patients in the ICU is shown in Table 3. Among patients with CA-LRTI and healthcare-associated LRTI, Streptococcus pneumoniae was over-represented among patients with CA-LRTI and healthcare-associated LRTI admitted to the ICU (accounting for $20 \%$ and $10.1 \%$ among patients admitted to the ICU versus $12.6 \%$ and $6.4 \%$ among patients in these cohort, $\mathrm{p}<0.001$ for both comparisons). Other typical community pathogens (Hemophillus influenza, Moraxella spp. and Bordatella spp.), gram negative bacteria and Staphylococcus aureus were not more prevalent among patients in the ICU when compared to their relative frequency in the entire cohort.

Table 3

Frequency of typical community pathogens and Staphylococcus aureus in respiratory tract cultures from hospitalized patients

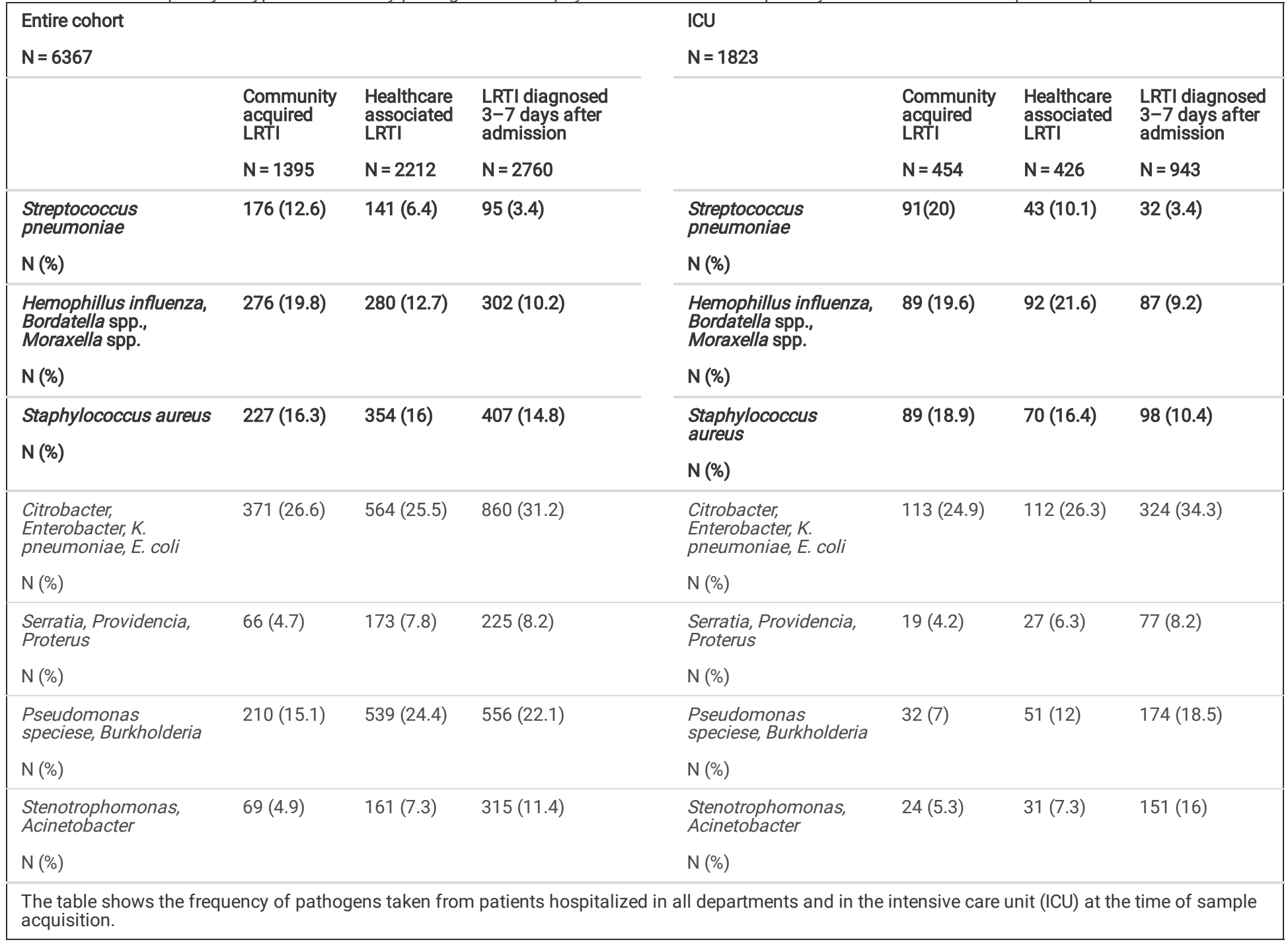

Antibiotic susceptibilities of various bacteria are shown in Table 4. Streptococcus pneumoniae was susceptible to penicillin in only around $50 \%$ of cases, and to erythromycin in $65 \%$ of cases. Streptococcus pneumoniae bacteria were susceptible in more than $95 \%$ of cases to fluoroquinolones (both ciprofloxacin and levofloxacin) and to 3rd generation cephalosporins (ceftriaxone). Other typical community-acquired pathogens (Hemophillus influenza, Moraxella spp., and Bordatella spp.) were also nearly universally susceptible to fluoroquinolones and 3rd generation cephalosporins, but about $25 \%$ were resistant to ampicillin. Gram negative bacteria were resistant to penicillins, penicillin and $\beta$-lactamase combinations, and 2nd generation cephalosporins in more than $50 \%$ of cases. Susceptibility rates to fluoroquinolones and 3rd generation cephalosporins were around $80 \%$ and $75 \%$ respectively (Table 4). 
Table 4

Antimicrobial susceptibilities of typical community pathogens and Staphylococcus aureus in L

\begin{tabular}{|c|c|c|c|c|c|c|c|c|c|}
\hline $\begin{array}{l}\text { Antibiotic } \\
\% \\
\text { Susceptible }\end{array}$ & $\begin{array}{l}\text { Penicillin } \\
\% \\
\text { susceptible }\end{array}$ & $\begin{array}{l}\text { Ampicillin } \\
\% \\
\text { susceptible }\end{array}$ & $\begin{array}{l}\text { Amoxicillin/Clavulonic } \\
\text { acid } \\
\text { \% susceptible }\end{array}$ & $\begin{array}{l}\text { Oxacillin } \\
\% \\
\text { susceptible }\end{array}$ & $\begin{array}{l}\text { Cefuroxime } \\
\% \\
\text { susceptible }\end{array}$ & $\begin{array}{l}\text { Ceftriaxone } \\
\% \\
\text { susceptible }\end{array}$ & $\begin{array}{l}\text { Ciprofloxacin } \\
\% \\
\text { susceptible }\end{array}$ & $\begin{array}{l}\text { Levofloxacin } \\
\% \\
\text { susceptible }\end{array}$ & $\begin{array}{l}\text { Erythron } \\
\% \\
\text { suscepti }\end{array}$ \\
\hline & \multicolumn{9}{|c|}{ Hemophillus, Bordatella, Moraxella } \\
\hline $\begin{array}{l}\text { Community } \\
N=271\end{array}$ & - & 74 & - & & 98.1 & 100 & 100 & 100 & - \\
\hline $\begin{array}{l}\text { Healthcare } \\
\text { associated } \\
N=242\end{array}$ & - & 75.6 & - & & 99.6 & 99.6 & 88.9 & 89.5 & - \\
\hline \multirow[t]{2}{*}{$\begin{array}{l}\text { Hospital N } \\
=284\end{array}$} & - & 71.9 & 97.3 & & 99.6 & 100 & 95.5 & 100 & - \\
\hline & \multicolumn{9}{|c|}{ Streptococcus pneumoniae } \\
\hline $\begin{array}{l}\text { Community } \\
N=176\end{array}$ & 59.1 & - & - & & - & 97.9 & - & 98.6 & 73.4 \\
\hline $\begin{array}{l}\mathrm{HCA} \\
\mathrm{N}=141\end{array}$ & 43.4 & - & - & & - & 95.3 & & 97.2 & 58.5 \\
\hline $\begin{array}{l}\text { Hospital } \\
\mathrm{N}=95\end{array}$ & 54.5 & - & - & & - & 97.2 & - & 100 & 62.3 \\
\hline & \multicolumn{9}{|c|}{ Citrobacter, Enterobacter, E. coli, Klebsiella } \\
\hline $\begin{array}{l}\text { Community } \\
\mathrm{N}=371\end{array}$ & - & 14.9 & 58.4 & & 69.2 & 84.3 & 89.9 & - & - \\
\hline $\begin{array}{l}\mathrm{HCA} \\
\mathrm{N}=564\end{array}$ & - & 8 & 47.9 & & 49.4 & 68.7 & 77.5 & - & - \\
\hline $\begin{array}{l}\text { Hospital } \\
N=860\end{array}$ & - & 6.7 & 49.8 & & 51.7 & 70.4 & 82.8 & - & - \\
\hline & Staphylococc & us aureus & & & & & & & \\
\hline $\begin{array}{l}\text { Community } \\
N=227\end{array}$ & 23.6 & - & - & 83.5 & - & - & 81.1 & 65.6 & 66.5 \\
\hline $\begin{array}{l}\mathrm{HCA} \\
\mathrm{N}=354\end{array}$ & 17.4 & - & - & 63.9 & - & - & 66.3 & - & 56.8 \\
\hline $\begin{array}{l}\text { Hospital } \\
N=403\end{array}$ & 25.9 & - & - & 66 & - & - & 69.4 & - & 57.6 \\
\hline
\end{tabular}

Table 5 presents the frequency of typical community pathogens, gram negative bacteria, and Staphylococcus aureus among patients who died within 14 days of obtaining the index culture. Streptococcus pneumoniae and gram negative bacteria were disproportionally represented among patients who died in the CALRTI group. Other typical community pathogens were disproportionally represented among patients who survived in all cohorts. 
Table 5

Fourteen-day mortality among patients with respiratory cultures of typical community pathogens, Staphylococcus aureus, and Gram-negative bacillii

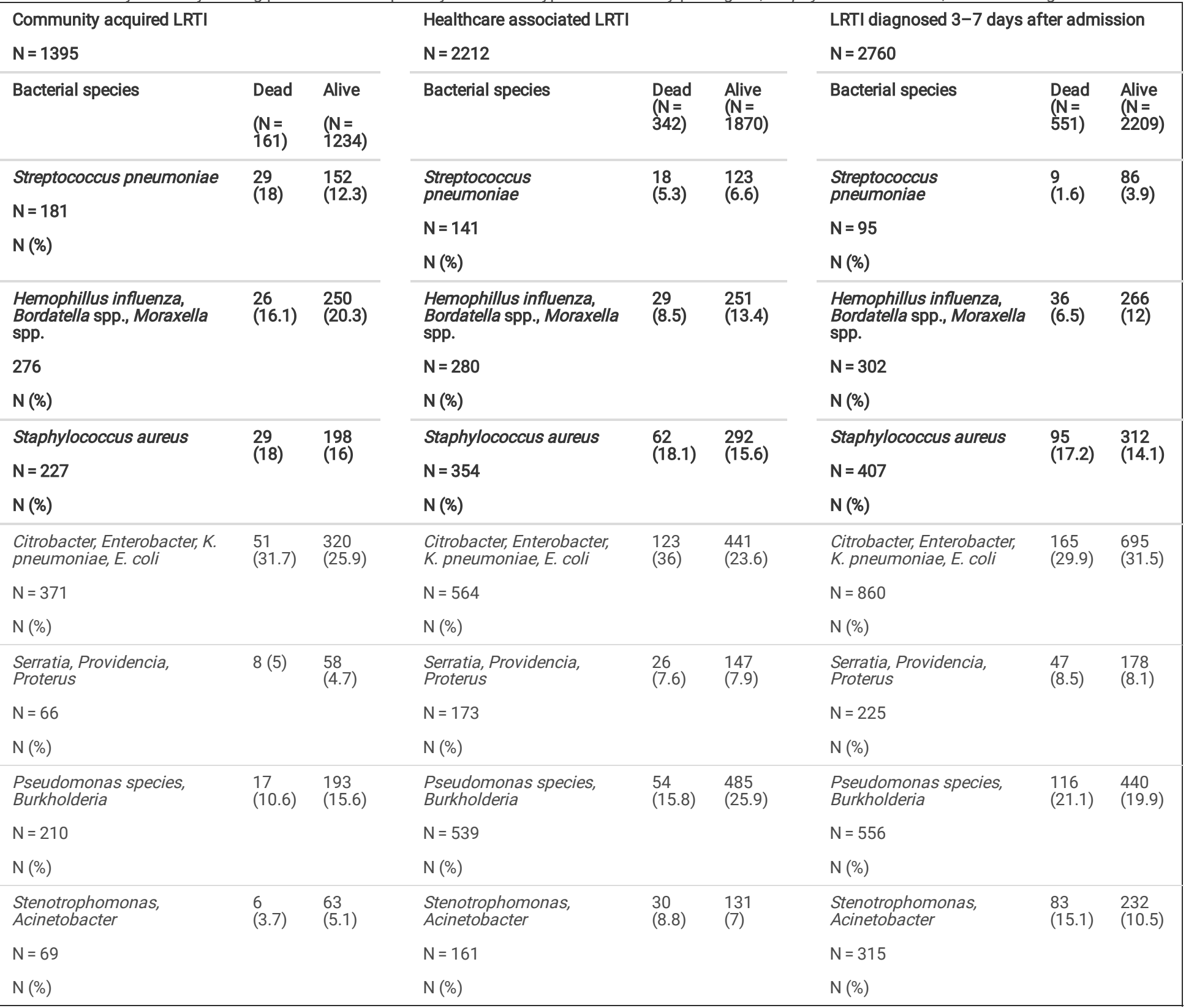

Factors associated with a 14 day mortality were analyzed in the entire cohort, including gram negative bacteria and typical community pathogens (Streptococcus pneumoniae, Hemophillus influenza, Moraxella spp., and Bordatella spp.). In the multivariable analysis, factors that were associated with increased mortality in the entire cohort were: age, admission to an ICU, admission to a surgical department, healthcare-associated or hospital-acquired infections (when compared to community acquired infections), and infections with Staphylococcus aureus, Enterobacteriaceae, or Stenotrophomonas and Acinetobacter baummannii (Tables $6 \mathrm{a}$ and $6 \mathrm{~b}$ ). 
Table 6

a: Predictors for 14-day mortality among hospitalized patients with LRTI

\begin{tabular}{|c|c|c|}
\hline Predictor & Adjusted OR (95\% Cl) & P-value \\
\hline Age* $^{*}$ & $1.026(1.02,1.032)$ & $<0.001$ \\
\hline \multicolumn{3}{|l|}{ Department } \\
\hline Internal medicine & 1 (reference) & \\
\hline Pediatric & $0.57(0.77,4.27)$ & 0.59 \\
\hline ICU & $3.37(2.34,4.85)$ & $<0.001$ \\
\hline Oncology-hematology & $0.53(0.07,3.97)$ & 0.53 \\
\hline Surgery-orthopedic & $2.48(1.34,4.60)$ & 0.004 \\
\hline \multicolumn{3}{|l|}{ Bacterial species } \\
\hline Streptococcus pneumonia & 1 (reference) & \\
\hline Gram negative bacteria associated with community-acquired infections ${ }^{\rrbracket}$ & $0.83(0.57,1.21)$ & 0.34 \\
\hline Staphylococcus aureus & $1.55(1.10,2.19)$ & 0.01 \\
\hline Non-fermenting gram negative bacteria ${ }^{€}$ & $1.06(0.75,1.49)$ & 0.75 \\
\hline Opportunistic bacteria common among ICU patients ${ }^{*}$ & $1.69(1.16,2.45)$ & 0.006 \\
\hline Enterobacteriacea & $1.39(1.001,1.93)$ & 0.048 \\
\hline Other gram negative bacteria ${ }^{£}$ & $1.23(0.83,1.82)$ & 0.30 \\
\hline \multicolumn{3}{|l|}{ Place of acquisition } \\
\hline Community acquired & 1 (reference) & \\
\hline Healthcare associated & $1.64(1.21,2.23)$ & 0.002 \\
\hline Hospital acquired & $3.08(2.28,4.15)$ & $<0.001$ \\
\hline \multicolumn{3}{|c|}{ OR, odds ratio; $\mathrm{Cl}$, confidence interval; ICU, intensive care unit; LRTI, lower respiratory tract infection } \\
\hline \multicolumn{3}{|l|}{${ }^{\circledR}$ Hemophillus influenza, Moraxella and Bordatella spp. } \\
\hline \multicolumn{3}{|l|}{$€$ Pseudomonas and Burkholderia spp } \\
\hline \multicolumn{3}{|l|}{$¥$ Stenotrophomonas and Acinetobacter spp } \\
\hline \multicolumn{3}{|l|}{ f Serratia, Providencia, and Proteus spp } \\
\hline *Increase for each year & & \\
\hline
\end{tabular}


Table 6

b: Predictors for 14-day mortality among patients who had respiratory cultures with typical community pathogens

\begin{tabular}{|c|c|c|}
\hline Predictor & Adjusted OR (95\% Cl) & P-value \\
\hline Age $^{\star}$ & $1.025(0.99,1.05)$ & 0.056 \\
\hline \multicolumn{3}{|l|}{ Charlson Comorbidity Index } \\
\hline Charlson Comorbidity Index = 0 (reference) & 1 & \\
\hline Charlson Comorbidity Index 1-4 & $0.40(0.14,1.15)$ & 0.09 \\
\hline Charlson Comorbidity Index $>4$ & $0.56(0.17,1.84)$ & 0.34 \\
\hline \multicolumn{3}{|l|}{ Department } \\
\hline Internal medicine & 1 (reference) & \\
\hline Children & $0.56(0.17,1.84)$ & 0.34 \\
\hline ICU & $2.23(1.44,3.55)$ & $<0.001$ \\
\hline Oncology-hematology & $1.74(0.63,4.78)$ & 0.28 \\
\hline Surgery-orthopedic & $1.87(1.07,3.26)$ & 0.03 \\
\hline \multicolumn{3}{|l|}{ Day culture was obtained } \\
\hline Community acquired & 1 (reference) & \\
\hline Healthcare associated & $0.88(0.08,9.32)$ & 0.92 \\
\hline Hospital acquired & $0.97(0.20,4.82)$ & 0.97 \\
\hline \multicolumn{3}{|c|}{ OR, odds ratio; $\mathrm{Cl}$, confidence interval; ICU, intensive care unit; } \\
\hline \multicolumn{3}{|l|}{ *Increase for each year } \\
\hline
\end{tabular}

In the multivariable analysis that included only typical community-acquired pathogens (Streptococcus pneumoniae, Hemophillus influenza, Moraxella spp., and Bordatella spp.), factors that were associated with an increased risk of mortality included ICU and surgical ward admission. Because of a strong interaction between the place of acquisition and the $\mathrm{CCl}$, the cohort was stratified into subgroups according to the place of acquisition and the multivariable analysis for a 14-day mortality was repeated. In the subgroup of healthcare-associated LRTI, a CCI between 1 and 4 , as well as a CCl greater than 4 were independent predictors for a 14-day mortality (OR 12.94, 95\% Cl 1.65, 101.15, p = 0.01; OR 14.84, 95\% Cl 1.70, 129.15, p=0.01, respectively). The CCI was not associated with a 14-day mortality in the other subgroups.

\section{Discussion}

This retrospective cohort study provides data on respiratory tract specimens of patients who were recently admitted to a hospital. Samples were divided into three groups: those taken within 48 hours of hospital admission from patients without known healthcare exposure; those taken within 48 hours of hospital admission from patients with known healthcare exposure; and samples taken 3-7 days after hospital admission. Gram negative bacteria were surprisingly the most common pathogens isolated in all of these cohorts. As expected, typical community-acquired pathogens (i.e. Streptococcus pneumoniae, Hemophillus influenza, Bordatella spp., and Moraxella spp.) were more frequently isolated among patients with community-acquired infections.

Although among young and relatively healthy patients, a larger proportion of samples had growth of typical community pathogens (Streptococcus pneumoniae, Hemophillus influenza, Bordatella spp., Moraxella spp.), in terms of absolute numbers the majority of these cases occurred in patients older than 40 years. Community-acquired LRTI cases caused by Streptococcus pneumoniae, but not other typical community pathogens, were more likely to be admitted to an ICU and to die.

Our results highlight the limited choice of available oral antibiotics for patients with CA-LRTIs. Primary care physicians often use penicillins with or without $\beta$ lactamase inhibitors, 2nd generation cephalosporins, macrolides, doxycycline, and fluoroquinolones for LRTIs. Typical community pathogens were nearly always susceptible to 3rd generation cephalosporins that are not universally available in an oral formulation, and to fluoroquinolones. Susceptibility to penicillin, ampicillin, and macrolides was consistently much lower among all study groups, and lower than those described in several previous European and North American studies [4, 11-13]. Gram negative bacteria, isolated mostly from frail elderly patients, were resistant to 2 nd generation cephalosporins, penicillins and $\beta$-lactamase inhibitors in more than $50 \%$ of cases. Although Hemophillus influenza, Bordatella spp., Moraxella spp. were nearly universally susceptible to amoxicillin and clavulanic acid, such combinations are unlikely to provide additional benefit over penicillins for the treatment of penicillinresistant Streptococcus pneumoniae, as resistance in these bacteria is mediated by mutations in the penicillin-binding protein rather than by the production of $\beta$-lactamases [14]. Other antibiotic agents may also not be adequate for the treatment of LRTI. For instance, trimethoprim sulphamethoxazole is not part of treatment guidelines. Furthermore, 2 nd generation cephalosporins in this study were active against only about $50 \%$ of gram negative bacteria, are probably less efficacious, and are mostly available in oral formulations with relatively low bioavailability $[15,16]$. 
The therapeutic implications of these data are not easy to determine, as many patients with CA-LRTI are treated empirically, and microbiologic data is either received late in the course of treatment or is unavailable altogether. However, it is likely that for the vast majority of younger patients with a low number of comorbid conditions, the likelihood of an adverse outcome from a CA-LRTI is very low, while the ecological price of antibiotic overuse is high [17]. Since CALRTIs are very common, resistance to administered antibiotics develops quickly [18]. Treatment with macrolides, 2nd generation cephalosporins, and penicillin and $\beta$-lactamase inhibitor combinations is likely to result in a good outcome in low-risk patients. Such low-risk patients who are infected with Streptococcus pneumoniae with intermediate resistance to penicillin can probably be adequately treated by higher doses of penicillin. Conversely, for patients who are at a higher risk of complications, i.e. older patients with multiple comorbid conditions, failure of a 1 st line antibiotic treatment administered in the community setting may result in increased use of 2 nd line antibiotics, and increased rates of hospital admission, mechanical ventilation, and death. A similar population includes older patients discharged from hospital to complete antibiotic treatment for an LRTI in the community. These patients nearly always have multiple risk factors for treatment failure and microbiologic data are usually unavailable. In such patients an initial or follow up treatment using penicillins with or without $\beta$-lactamase inhibitors, macrolides, or 2nd generation cephalosporins may be inadequate. Fluoroquinolones, and more specifically the so called "respiratory" fluoroquinolones, provide excellent coverage according to our data and those of previous studies [19-21]. The use of fluoroquinolones, however, has several important drawbacks for patients with significant comorbidities. Namely, they are associated with an increased risk of Closridioides difficile associated diarrhea and probably more so than most other antibiotics [22]; they can cause QT prolongation that may lead to arrhythmias; and their use is associated with tendinopathies, peripheral neuropathy, and rupture of aortic aneurysms [22]. Treatment with fluoroquinolones quickly leads to colonization with resistant pathogens in the treated patients $[18,23]$, and increases the risk of fluoroquinolones resistance in certain geographic areas even in the absence of personal antibiotic use [24].

Improving diagnostics with the use of molecular tools for identification of respiratory pathogens and / or their resistance genes may facilitate antibiotic choice in the near future. In addition, newer antibiotics such as lefamulin, and oral formulations of 3rd generation cephalosporins (i.e. cefditoren pivoxil or cefpodoxime) may prove useful for high-risk patients with relative contraindications to fluoroquinolones.

There are several important limitations for this study. The data are retrospective, and are based on microbiological sampling results. Occasional patients with no active infections could have been included, although RHCC policy is that respiratory tract samples are taken only for patients with suspected infections. Conversely, samples may not have been obtained for various reasons from patients with clinically significant LRTI. We may have missed some CA-LRTI cases caused by less common pathogens. Empirical treatment, however, should be based on the antibiotic susceptibility profile, safety, ecological cost and economic cost of specific agents, and targeted towards the most common community pathogens. Susceptibility tests were performed according to CLSI requirements. Therefore, there are some gaps in potentially useful data, i.e. Streptococcus pneumoniae susceptibility rates to 2 nd generation cephalosporins.

\section{Conclusions}

Our data shows that gram negative bacteria are very common among patients admitted because of a LRTI, and typical community acquired pathogens (Streptococcus pneumoniae, Hemophillus influenza, Bordatella spp., Moraxella spp.) are especially common among older patients with multiple comorbidities. The isolation of these bacteria in respiratory tract samples is associated with appreciable rates of ICU admission and 14-day mortality rates. The susceptibility pattern of these community pathogens suggests that for high-risk patients, the choice of effective oral antibiotic agents is limited. Both improvements in diagnostic procedures aimed at providing targeted therapy and newer oral antibiotic formulations are needed.

\section{List Of Abbreviations}

CA-LRTI - community-acquired lower respiratory tract infections

$\mathrm{CCl}$ - CharlsonComorbidity Index

CLSI - Clinical and Laboratory Standards Institute

ICU - intensive care unit

IQR - interquartile ranges

LTCF - long-term care facility

LRTI - lower respiratory tract infections

RHCC - Rambam Health Care Campus

\section{Declarations}

Competing interests: Neuberger Ami has received writing honoraria from Taro Pharmaceuticals Industries Ltd.

Funding: This work was supported by a research grant from Taro Pharmaceuticals Industries Ltd.

Authors contributions: $\mathrm{OH}$ - data analysis, writing of manuscript. ARB - data analysis, review of manuscript, AN - study concepts, writing of manuscript

Ethics approval and consent to participate: This study protocol was exempted for review by the Institutional ReviewBoard of the Rambam Health Care Center according to the exemptioncriteria. In this study, anonymized and deidentified information was used forthe analyses; thus, informed consent was not required.

Page 10/12 
Consent for publication:Not applicable.

Availability of data and materials:The datasets used and/or analyzed during the current study are available from the corresponding author on reasonable request.

Acknowledgement: We would like to thank Dr. Ruth Moont for her assistance in reviewing, editing, and preparing the manuscript.

\section{References}

1. Walter JM, Wunderink RG. Severe Respiratory Viral Infections: New Evidence and Changing Paradigms. Infect Dis Clin North Am. 2017;31(3):455-74. doi:10.1016/j.idc.2017.05.004.

2. Kocsis B, Szabo D. New treatment options for lower respiratory tract infections. Expert Opin Pharmacother. 2017;18(13):1345-55. doi:10.1080/14656566.2017.1363179.

3. Groeneveld GH, van 't Wout JW, Aarts NJ, et al. Prediction model for pneumonia in primary care patients with an acute respiratory tract infection: role of symptoms, signs, and biomarkers. BMC Infect Dis. 2019;19(1):976. doi:10.1186/s12879-019-4611-1.

4. Torres A, Blasi F, Peetermans WE, Viegi G, Welte T. The aetiology and antibiotic management of community-acquired pneumonia in adults in Europe: a literature review. Eur J Clin Microbiol Infect Dis. 2014;33(7):1065-79. doi:10.1007/s10096-014-2067-1.

5. Jiang LX, Ren HY, Zhou HJ, et al. Simultaneous Detection of 13 Key Bacterial Respiratory Pathogens by Combination of Multiplex PCR and Capillary Electrophoresis. Biomed Environ Sci. 2017;30(8):549-61. doi:10.3967/bes2017.074.

6. Ramirez JA, Ahkee S, Tolentino A, Miller RD, Summersgill JT. Diagnosis of Legionella pneumophila, Mycoplasma pneumoniae, or Chlamydia pneumoniae lower respiratory infection using the polymerase chain reaction on a single throat swab specimen. Diagn Microbiol Infect Dis. 1996;24(1):7-14. doi:10.1016/0732-8893(95)00254-5.

7. von Baum H, Welte T, Marre R, Suttorp N, Ewig S. CAPNETZ study group. Community-acquired pneumonia through Enterobacteriaceae and Pseudomonas aeruginosa: Diagnosis, incidence and predictors. Eur Respir J. 2010;35(3):598-605. doi:10.1183/09031936.00091809.

8. El-Solh AA, Sikka P, Ramadan F, Davies J. Etiology of severe pneumonia in the very elderly. Am J Respir Crit Care Med. 2001;163(3 Pt 1):645-51. doi:10.1164/ajrccm.163.3.2005075.

9. Verduin CM, Hol C, Fleer A, van Dijk H, van Belkum A. Moraxella catarrhalis: from emerging to established pathogen. Clin Microbiol Rev. 2002;15(1):12544. doi:10.1128/cmr.15.1.125-144.2002.

10. Marchello C, Dale AP, Thai TN, Han DS, Ebell MH. Prevalence of Atypical Pathogens in Patients With Cough and Community-Acquired Pneumonia: A MetaAnalysis. Ann Fam Med. 2016;14(6):552-66. doi:10.1370/afm.1993.

11. leven $\mathrm{M}$, Coenen $\mathrm{S}$, Loens $\mathrm{K}$, et al. Aetiology of lower respiratory tract infection in adults in primary care: a prospective study in 11 European countries. Clin Microbiol Infect. 2018;24(11):1158-63. doi:10.1016/j.cmi.2018.02.004.

12. Gadsby NJ, Russell CD, McHugh MP, et al. Comprehensive Molecular Testing for Respiratory Pathogens in Community-Acquired Pneumonia. Clin Infect Dis. 2016;62(7):817-23. doi:10.1093/cid/civ1214.

13. Bartlett JG. Diagnostic tests for agents of community-acquired pneumonia. Clin Infect Dis. 2011;52(Suppl 4):296-304. doi:10.1093/cid/cir045.

14. Smith AM, Klugman KP. Alterations in penicillin-binding protein 2B from penicillin-resistant wild-type strains of Streptococcus pneumoniae. Antimicrob Agents Chemother. 1995;39(4):859-67. doi:10.1128/aac.39.4.859.

15. Yu VL, Chiou CC, Feldman C, et al. An international prospective study of pneumococcal bacteremia: correlation with in vitro resistance, antibiotics administered, and clinical outcome. Clin Infect Dis. 2003;37(2):230-7. doi:10.1086/377534.

16. Williams PE, Harding SM. The absolute bioavailability of oral cefuroxime axetil in male and female volunteers after fasting and after food. $J$ Antimicrob Chemother. 1984;13(2):191-6. doi:10.1093/jac/13.2.191.

17. Coxeter P, Del Mar CB, McGregor L, Beller EM, Hoffmann TC. Interventions to facilitate shared decision making to address antibiotic use for acute respiratory infections in primary care. Cochrane Database Syst Rev. 2015;2015(11):CD010907. doi:10.1002/14651858.CD010907.pub2. Published 2015 Nov 12.

18. Costelloe C, Metcalfe C, Lovering A, Mant D, Hay AD. Effect of antibiotic prescribing in primary care on antimicrobial resistance in individual patients: systematic review and meta-analysis. BMJ. 2010;340:c2096. Published 2010 May 18. doi:10.1136/bmj.c2096.

19. Jones RN, Jacobs MR, Sader HS. Evolving trends in Streptococcus pneumoniae resistance: implications for therapy of community-acquired bacterial pneumonia. Int J Antimicrob Agents. 2010;36(3):197-204. doi:10.1016/j.ijantimicag.2010.04.013.

20. Naucler P, Henriques-Normark B, Hedlund J, Galanis I, Granath F, Örtqvist Å. The changing epidemiology of community-acquired pneumonia: nationwide register-based study in Sweden. J Intern Med. 2019;286(6):689-701. doi:10.1111/joim.12956.

21. Wunderink RG, Yin Y. Antibiotic Resistance in Community-Acquired Pneumonia Pathogens. Semin Respir Crit Care Med. 2016;37(6):829-38. doi:10.1055/s-0036-1593753.

22. 10.1093 /ofid/of $x 171$

Guh AY, Adkins SH, Li Q, et al. Risk Factors for Community-Associated Clostridium difficile Infection in Adults: A Case-Control Study. Open Forum Infect Dis. 2017;4(4):ofx171. Published 2017 Oct 26. doi:10.1093/ofid/ofx171.

23. Stewardson AJ, Vervoort J, Adriaenssens N, et al. Effect of outpatient antibiotics for urinary tract infections on antimicrobial resistance among commensal Enterobacteriaceae: a multinational prospective cohort study. Clin Microbiol Infect. 2018;24(9):972-9. doi:10.1016/j.cmi.2017.12.026. 
24. Low M, Neuberger A, Hooton TM, et al. Association between urinary community-acquired fluoroquinolone-resistant Escherichia coli and neighbourhood antibiotic consumption: a population-based case-control study. Lancet Infect Dis. 2019;19(4):419-28. doi:10.1016/S1473-3099(18)30676-5.

\section{Figures}

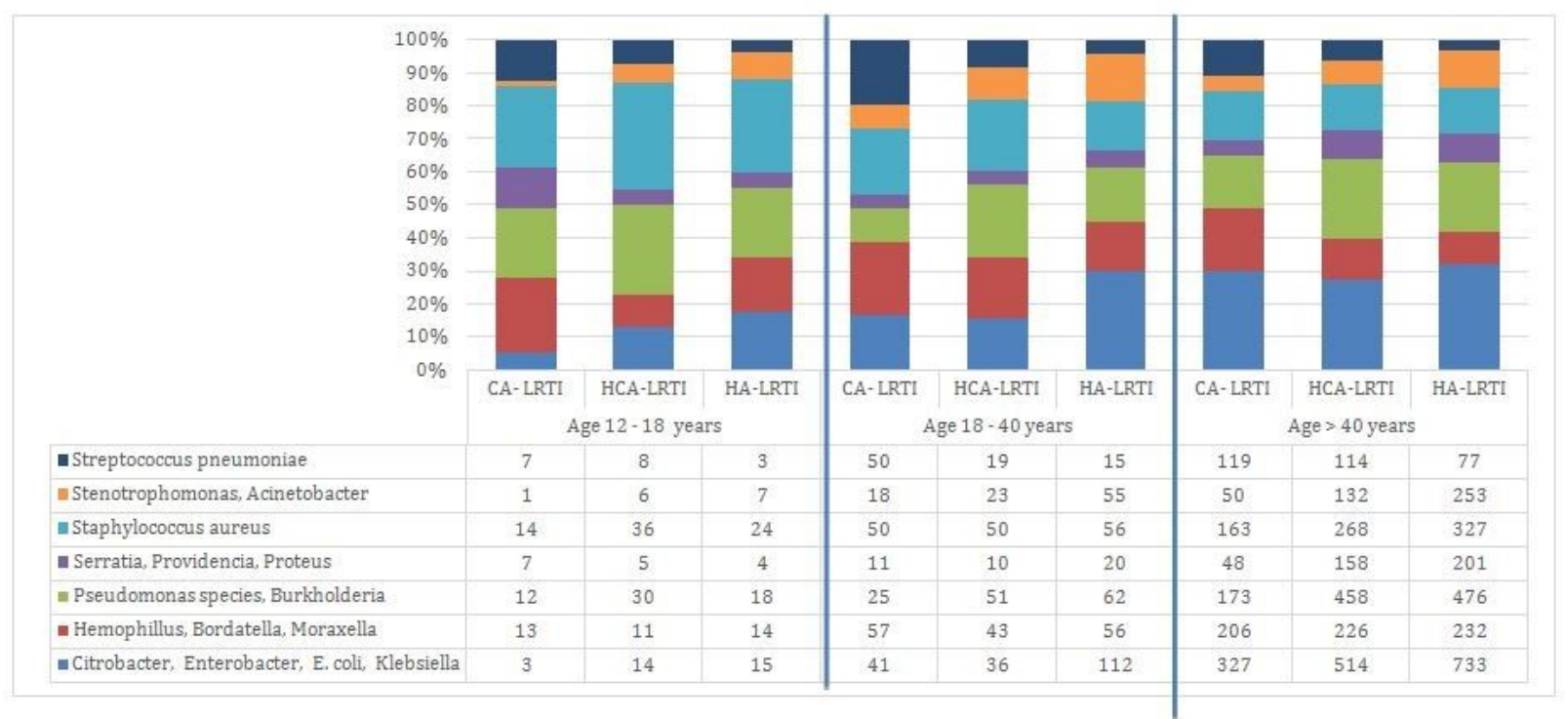

CA. compunity-acquired. LRTI. lower respiratory tract infection. HCA. healthcare-asseciated. HA hospital-acquired. Community-acquired LRTI consist of samples taken within 0-2 days of hospital admission. Healthcare-associated LRTI consist of samples taken within 0-2 days of hospital admission and with recent healthcare exposure. Hospital-acquired LRTI consist of samples taken within 3-7 days of hospital admission.

\section{Figure 1}

Frequency of bacteria isolated in respiratory tract cultures taken from hospitalized patients by age groups



CA. community-acquired. IRTI, lower respiratory tract infection: HCA helthcare-associated. HA hospital-acquired. Community-acquired LRTI consist of samples taken within 0-2 days of hospital admission. Healthcare-associated LRTI consist of samples taken within 0-2 days of hospital admission and with recent healthcare exposure. Hospital-acauired LRTI consist of samples taken within 3-7 days of hospital admission.

\section{Figure 2}

Frequency of bacteria isolated in respiratory tract cultures taken from patients by Charlson Comorbidity Index 